City University of New York (CUNY) CUNY Academic Works

1986

\title{
General Results on Glueball Masses in QCD
}

\author{
I. J. Muzinich \\ University of California, Santa Barbara \\ V. Parameswaran Nair \\ City College of New York
}

\section{How does access to this work benefit you? Let us know!}

More information about this work at: https://academicworks.cuny.edu/cc_pubs/465

Discover additional works at: https://academicworks.cuny.edu

This work is made publicly available by the City University of New York (CUNY).

Contact: AcademicWorks@cuny.edu 


\title{
GENERAL RESULTS ON GLUEBALL MASSES IN QCD
}

\author{
I.J. MUZINICH and V.P. NAIR \\ Institute for Theoretical Physics, University of California, Santa Barbara, CA 93106, USA
}

Received 6 May 1986

\begin{abstract}
A number of authors have investigated mass inequalities for mesons and baryons in QCD. These provide rigorous nonperturbative constraints on the mass spectrum. Similar inequalities for glueballs are investigated. For nonzero spin $J$, in the large $-N_{\mathrm{c}}$ approximation, $m_{J^{-}} \geqslant m_{J^{+}}$is found. (For $J=0$, the existence of a gluon condensate can modify this statement.) There are also constraints on how fast $m_{J}$ can grow with $J$. For example, for $m_{J}=a+b J^{\alpha}, 0 \leqslant \alpha \leqslant 1$ is found, a result consistent with Regge behaviour.
\end{abstract}

The existence of glueballs is expected to be, qualitatively, one of the most significant features of QCD. Nevertheless our understanding of the spectrum of glueballs is very limited. Although there are a number of different QCD based models for glueballs $[1,2]^{\ddagger 1}$, even broad general questions, such as whether (and if so, how closely) they lie on Regge trajectories, do not seem to have model-independent answers. The planar graph approximation (or the large $-N_{\mathrm{c}}$ approximation) of QCD does suggest a Regge-type spectrum [6] ${ }^{\ddagger 2}$ but a stronger result is still lacking. It is of some interest therefore to study inequalities on glueball masses. Although such inequalities are generally not stringent enough to do quantitative physics, they are modelindependent consequences of very general features of $Q C D$ and at least give constraints on various $Q C D$ based models for glueballs.

Mass inequalities have been derived and successfully applied to the flavor non-singlet sector of QCD viz. mesons and baryons by a number of authors $[8-10]$. The central ingredient in their derivation is positivity of the functional measure of the euclidean path integral and the existence of a mass gap. In particular Weingarten and Witten and others have derived general results on the mass spectrum of QCD. Perhaps the most striking

$\$ 1$ For potential models see ref. [3], for models based on the scale anomaly see ref. [4], for models based on dual superconductivity see ref. [5].

$\neq 2$ For a review see ref. [7]. conclusion is $m_{\chi} \geqslant m_{\pi}$, where $m_{\chi}$ is the mass of any flavor non-singlet with the quantum numbers of a quark bilinear and $m_{\pi}$ is the pion mass.

Witten and Vafa [8] have further extended the Weingarten result to include results of the generic type $2 m_{A \bar{B}} \geqslant m_{A \bar{A}}+m_{B \bar{B}}$ where $A$ and $B$ refer to different quark flavors and the positivity of the electromagnetic mass shift of the pion. Similar results have been established for the quark model sector based on potential theory by Nussinov and Lieb [10]. In particular the relation between baryon and boson masses viz.

$m_{\text {baryon }}=\frac{3}{2} m_{\text {boson }}$ of the additive quark model is obtained.

In this paper we consider inequalities for correlation functions involving color singlet composite operators which are polynomials of the field strength tensor and its covariant derivatives. Again, these inequalities depend only on the positivity of the euclidean functional measure. The correlation functions fall off exponentially at large separations at a rate given by the mass of the lowest lying glueball of the appropriate quantum numbers. Thus, with the assumption of a mass gap, the correlation inequalities translate into mass inequalities. The composite operators act as interpolating fields for glueballs in the LSZ sense in the Heisenberg picture of conventional field theory.

In general the masses are expected to increase with the spin $J$ or dimension $d$ of the interpolating field. For a mass formula of the type $m=a+b d^{\alpha}$ (or $a+$ $b J^{\alpha}$ ), we find $\alpha \leqslant 1$. This is consistent with Regge be- 
haviour $\left(\alpha=\frac{1}{2}\right)$. Also generically we have $m_{J^{-}} \geqslant m_{J^{+}}$ where \pm refer to even and odd parities ${ }^{\ddagger 3}$. The $0^{++}$ channel can be an exception to this because of the possibility of a vacuum expectation for $F_{\mu \nu}^{a} F_{\mu \nu}^{a}$, which can be interpreted as a vacuum condensate in which case trivially $m_{0^{-}} \geqslant m_{0^{+}}$. However the physical glueball is the fluctuation of $F^{2}$ from its vacuum value. We have no constraint in this case. The results for spins higher than zero are expected to hold in the large- $N_{\mathrm{c}}$ approximation.

We start our discussion with some kinematical remarks. The local operators can be constructed as polynominals of the field strength tensor $F_{\mu \nu}^{a}$ and the quark fields. Some simple examples are $\operatorname{tr}\left(F_{\mu \nu} F_{\mu \nu}\right)$ which creates $0^{++}$states, $\operatorname{tr}\left(F_{\mu \nu} \widetilde{F}_{\mu \nu}\right)$ for $0^{-+}$states and $\theta_{\alpha \beta}=$ $\operatorname{tr}\left(F_{\alpha \mu} F_{\mu \beta}-\frac{1}{4} \delta_{\alpha \beta} F^{2}\right)$ for $2^{++}$states. (Here $F_{\mu \nu}=$ $F_{\mu \nu}^{a} t^{a}, t^{a}$ are color matrices and $\widetilde{F}_{\mu \nu}=\frac{1}{2} \epsilon_{\mu \nu \alpha \beta} F_{\alpha \beta}$.) One can also have mixtures with quarks, e.g. $F_{\mu \nu}^{a} \overline{\mathrm{q}} t^{a} \mathrm{q}$ which gives $1^{ \pm-}$. Examples of operators of dimension 6 are $\operatorname{tr}\left[F_{\mu \nu}, F_{\lambda \sigma}\right] F_{\alpha \beta}$ which gives $J=0,1,2,3$, with $C=+1$ and $\operatorname{tr}\left\{F_{\mu \nu}, F_{\lambda \sigma}\right\} F_{\alpha \beta}$ for $J=0,1,2,3$ with $C$ $=-1$. Needless to say, these are interpolating fields in the LSZ sense and so operators of the same quantum numbers can mix. A detailed kinematic analysis of glueball quantum numbers based on acceptable interpolating fields has recently been presented [11].

The measure of integration, in euclidean space, is given for QCD at $\theta=0$ by

$$
\begin{gathered}
\mathrm{d} \mu(A)=\frac{1}{Z} \prod_{x, \mu, a}\left[\mathrm{~d} A_{\mu}^{a}(x)\right] \operatorname{det}(\not D+m) \\
X \exp \left(-\frac{1}{4 g^{2}} \int F_{\mu \nu}^{a} F_{\mu \nu}^{a} \mathrm{~d}^{4} x\right) .
\end{gathered}
$$

$Z$ is a normalization factor. In a vector-like theory such as $\mathrm{OCD}$, the fermion determinant is of the form $\Pi_{n}\left(\mathrm{~m}^{2}\right.$ $+\lambda_{n}^{2}$ ) where the $\lambda_{n}$ are the (real) eigenvalues of $-\mathrm{i} \not$, i.e., $\not \psi_{n}=\mathrm{i} \lambda_{n} \psi_{n}$. The measure is thus manifest ly positive. Since the measure is positive, inequalities which hold for each $A_{\mu}^{a}$ are also valid after integration over $A_{\mu}^{a}$.

The correlation functions we are interested in are of the form

$\left\langle P_{I}(x) P_{J}(0)\right\rangle=\int \mathrm{d} \mu(A) P_{I}(x) P_{J}(0)$,

$\neq 3$ The notation for the glueball state is $J P C$, where $J$ is the spin, $P$ denotes parity and $C$ denotes charge conjugation. $C^{-1} F_{\mu \nu} C=-F_{\mu \nu}^{\mathrm{T}}$. where $P_{I}(x)$ is a local gauge invariant operator; $I, J$ are generic indices for the Lorentz tensor structure of $P$. For the pure glueball sector, $P(x)$ are also flavor singlets.

In order to define such formal correlation functions, one has to have a regulator which preserves the Lorentz covariance properties of the composite operators. We shall not give a detailed analysis of how this can be done, but indicate briefly how the random lattice formulation of gauge theories would provide such a regulator [12]. The measure of integration (1) involves integration over potentials at each spacetime point. Since the action is gauge invariant, the integration ove the pure gauge directions of the potential gives a factor of the group volume at each point. This would diverge for an infinite number of points and so we have to put the theory on some kind of lattice (with a finite number of points) to make (1) well defined. A possibl way to preserve Lorentz covariance properties would be to use a random lattice. Composite operator correlations on a random lattice can be generated as follows. One considers the Wilson loop operators $W\left(\mathrm{C}_{1}\right)$, $W\left(C_{2}\right)$ defined for curves $C_{1}$ and $C_{2}$. The plaquette bounded by $\mathrm{C}_{1}$ is chosen to be rotated relative to the plaquette corresponding to $\mathrm{C}_{2}$ by $R$, an element of the four-dimensional rotation group. The functional average of the product $W\left(\mathrm{C}_{1}\right) W\left(\mathrm{C}_{2}\right)$ is evaluated as a function of the separation of the two plaquettes and $R$ and various spins are projected out using the harmonics of the rotation group. In the naive continuum limit such correlations tend to averages such as (2). The Wilson loop operators in the above set of statements are only an example. One can also use other lattice operators to define a large number of composit operator correlations. The correlations we consider are physical renormalization group invariant quantities and the results are not expected to depend on how the theory is renormalized.

One can make a spectral decomposition of the twopoint function (2).

$$
\begin{aligned}
& \left\langle P_{I}(x) P_{J}(0)\right\rangle \\
& \quad=\int \mathrm{d} m^{2} \sum_{n} \rho_{n}\left(m^{2}\right) D_{I J}^{(n)}\left(-\mathrm{i} \partial_{x}\right) \Delta_{\mathrm{F}}\left(x, m^{2}\right),
\end{aligned}
$$

where $\Delta_{F}\left(x, m^{2}\right)$ is the propagator of the free field theory, $\rho_{n}\left(m^{2}\right)$ is the spectral function whose region of support gives the mass spectrum in the $I J$ channel, 
and $D_{I J}^{(n)}$ is a polynomial which gives the correct Lorentz decomposition. The idea of confinement supports the assumption of a mass gap, i.e., $\rho\left(\mathrm{m}^{2}\right)$ $=0$ for $m^{2}<m_{0}^{2} \neq 0 . m_{0}^{2}$ is the mass of the lowest lying glueball with the quantum numbers of the operator $P_{I}(x)$. We assume that $m_{0}^{2}$ is part of the discrete spectrum; for example, for $\mathrm{SU}\left(N_{\mathrm{c}}\right), N_{\mathrm{c}} \rightarrow \infty$ this is the case. Since all the quarks carry nonzero (current algebra) masses, we do not expect any flavor singlet massless particles to form a multiparticle continuum with glueballs. With these assumptions, the large separation behavior of correlation functions will be given by

$\left\langle P_{I}(x) P_{J}(0)\right\rangle \simeq c \exp \left(-m_{0}|x|\right)$

(as $|x| \rightarrow \infty$ and for $\left\langle P_{I}(x)\right\rangle=0$ ).

We shall consider a typical inequality before going to the general case. Define

$\phi^{a A}(x)=F_{\mu \nu}^{a}(x) M_{\mu \nu}^{A}, \quad \chi^{a A}(x)=\tilde{F}_{\mu \nu}^{a}(x) M_{\mu \nu}^{A}$,

where $A$ denotes a set of spacetime indices and $M_{\mu \nu}^{A}$ is a tensor to be specified. Define $U$ and $V$ by

$U=\phi^{a A}(x) \phi^{b B}(0), \quad V=\chi^{a A}(x) \chi^{b B}(0)$.

The Cauchy-Schwarz inequality $\left|\operatorname{Tr} U^{\dagger} V\right|^{2} \leqslant \operatorname{Tr} U^{\dagger} U$ $\operatorname{Tr} V^{\dagger} V$ gives

$\left(\phi^{2}(x) \phi^{2}(0) \chi^{2}(x) \chi^{2}(0)\right) \geqslant|\phi \cdot \chi(x) \phi \cdot \chi(0)|^{2}$, .

where $\phi^{2}=\phi^{a A} \phi^{a A}$, etc. We now choose $M_{\mu \nu}^{a}$ to be $\delta_{\mu}^{\alpha} p_{\nu}^{\beta}$. The inequality becomes, with $\phi_{\alpha \beta}=F_{\mu \alpha}^{a} F_{\mu \beta}^{a}$, $\chi_{\alpha \beta}=F_{\mu \alpha}^{a} \widetilde{F}_{\mu \beta}^{a}$,

$\left|\phi_{\alpha \beta}(x) p_{\alpha \beta} \phi_{\mu \nu}(0) p_{\mu \nu}\right|^{2} \geqslant\left|\chi_{\alpha \beta}(x) p_{\alpha \beta} \chi_{\mu \nu}(0) p_{\mu \nu}\right|^{2}$.

The operator $\phi_{\alpha \beta}$ gives $2^{++}$and $0^{++}$glueballs while $\chi_{\alpha \beta}$ gives $2^{-+}$and $0^{-+}$glueballs. We take $p_{\mu \nu}$ to be zero for all $\mu, \nu$ except for one offdiagonal choice corresponding to one of the spin-two states e.g. $\mu, \nu=i j=$ 2,3 . The inequality $(8)$ gives

$\left|\phi_{i j}(x) \phi_{i j}(0)\right|^{2} \geqslant\left|\chi_{i j}(x) \chi_{i j}(0)\right|^{2}$

(no summation on $i, j$ ). Upon averaging over gluon configurations as in eq. (2) and using eq. (4) we obtain $m_{2^{++}} \leqslant m_{2^{-+}}$. By choosing $p_{\mu \nu}=\delta_{\mu \nu}$ we find $\left\langle F^{2}(x) F^{2}(0)\right\rangle \geqslant\langle F \widetilde{F}(x) F \tilde{F}(0)\rangle$.

At large separations, $\left\langle F^{2}(x) F^{2}(0)\right\rangle=\left\langle F^{2}\right\rangle^{2}$ if there is a $\operatorname{VEV}\left\langle F^{2}\right\rangle \neq 0$. The inequality is then trivially satisfied for any $m_{0^{-+}}>0$. If $\left\langle F^{2}\right\rangle$ is zero, the inequality (10) leads to $m_{0^{-+}} \geqslant m_{0^{++}}$. A non zero $\left\langle F^{2}\right\rangle$ could also be interpreted as $m_{0^{+}}=0$. However if $\left\langle F^{2}\right\rangle \neq 0$, the physical glueball must be defined in terms of the connected part of $\left\langle F^{2}(x) F^{2}(0)\right\rangle$ and the corresponding lowest mass $m_{0}+$ is not constrained in any way by (10).

Another useful inequality is obtained by taking $U=$ $F_{\alpha i}^{a}(x) F_{\beta i}^{b}(0), V=F_{\alpha k}^{a}(x) F_{\beta k}^{b}(0)$ (no summation over $i$ or $k$ ). This leads to

$\left|\phi_{i k}(x) \phi_{i k}(0)\right|^{2} \leqslant \phi_{i i}(x) \phi_{i i}(0) \phi_{k k}(x) \phi_{k k}(0)$.

Choosing $U=F_{\alpha i}^{a}(x) F_{\beta k}^{b}(0)(i, k$ fixed) we obtain from $\operatorname{Tr} U^{\dagger} U \geqslant 0, \phi_{i i}(x) \phi_{k k}(0) \geqslant 0$. The right-hand side of (11) can be thus extended to a full sum giving

$\left|\phi_{i k}(x) \phi_{i k}(0)\right|^{2} \leqslant\left|F^{2}(x) F^{2}(0)\right|^{2}$.

Upon averaging over gluon configurations this leads to the inequality $m_{2^{++}} \geqslant m_{0^{++}}$. Again if $\left\langle F^{2}\right\rangle \neq 0$, this inequality is trivial with $m_{0^{++}}=0$. For $M_{0^{++}}$defined in terms of the connected part of $\left\langle F^{2}(x) F^{2}(0)\right\rangle$, we have no constraint vis à vis $m_{2++}$. However if there is an independent estimate of $M_{0^{++}}$giving $M_{0^{++}}>m_{2++}$ (which has been suggested by lattice gauge theory Monte Carlo studies [13]), the inequality (12) will lead to the conclusion that $\left\langle F^{2}\right\rangle \neq 0$. Experimental data that may be interpreted as evidence for spin-2 glueballs has been obtained for the reaction $\pi^{-} \mathrm{p} \rightarrow$ $\phi \phi \mathrm{n}[14]$. A comment about the operator $\phi_{\mu \nu}$ is in order. $\phi_{\mu \nu}$ contains a $1^{-+}$piece viz. $\phi_{0 i}$. However, by Lorentz covariance, it cannot create $1^{-+}$glueballs. $\phi_{\mu \nu}$ is conserved, up to gradients of the scalar operator $F^{2}$, and the amplitude $\left\langle\phi_{\mu \nu}(x) \phi_{\alpha \beta}(0)\right\rangle$ therefore contains only two Lorentz invariant terms, one each for spins zero and two. The lowest dimensional operator built up of $F_{\mu \nu}$ 's which gives $1^{-+}$involves three $F_{\mu \nu}$ 's [11].

We now turn to the case of operators of arbitrary dimension. In this case it is easier to use the two component spinor notation for SL $(2, \mathbf{C})$ tensors. By use of $\epsilon_{a b}$ and $\epsilon_{\dot{a} \dot{b}}$, the local operators we construct can be reduced to irreducible tensors. An irreducible tensor is a multispinor of $2 k$ undotted indices and $2 n$ dotted indices, symmetric in each set. These are $\mathrm{SU}(2) X$ $\mathrm{SU}(2)$ indices since we are in euclidean space. Upon continuation to Minkowski space, they go over to corresponding $\operatorname{SL}(2, \mathrm{C})$ indices. Consider $A_{a b c . .}^{(a)}$ with 
$2 J$ undotted indices. The upper $(a)$ denotes a generic color index not restricted to the adjoint representation. Define $U$ and $V$ as follows:

$$
\begin{aligned}
& U=A_{a b c \ldots}^{(a)}(x) M_{a b c \ldots}^{A} A_{\dot{p} \dot{q} \dot{r} \ldots}^{(b)}(0) M_{\dot{p} \dot{q} \dot{r} \ldots}^{(B)}, \\
& V=B^{(a)}(x) B^{(b)}(0) \delta^{A B},
\end{aligned}
$$

where $B^{(a)}(x)$ is a scalar. $M_{a b c \ldots}^{A}$ is again to be specified. Denoting $M_{a b c \ldots . .}^{a} M_{a \dot{a} b \dot{c} \ldots . .}^{a}=P_{a b c \ldots, a} \dot{a} \dot{b} \dot{c} \ldots$, the CauchySchwarz inequality gives

$$
\begin{aligned}
\mid B & \left.\cdot A_{a b c \ldots(0) P_{a b c \ldots, a} \dot{b} \dot{c} \dot{c} \ldots} B \cdot A_{\dot{a} \dot{b} \dot{c} \ldots}(x)\right|^{2} \\
& \leqslant f\left(B^{2}(x) B^{2}(0)\right) \\
& \times\left(A_{a b c \ldots a \dot{a} \dot{b} \dot{c} \ldots}^{2}(x) P_{a b c \ldots, \dot{a} \dot{b} \dot{c} \ldots}\right. \\
& \left.\times A_{p q r \ldots, \dot{p} \dot{q} \dot{r} \ldots}^{2}(0) P_{p q r \ldots, \dot{p} \dot{q} \dot{r} \ldots}\right) .
\end{aligned}
$$

$f=\delta^{A B} \delta^{B A}, A^{2}$ and $B \cdot A$ indicate contraction with respect to color indices. In the intermediate states on the left side we get states of spin $J$, e.g., $a b c \ldots=111 \ldots$. On the right-hand side the intermediate states have $J_{3}$ $=0$. Since the left-hand side of eq. (14) is linear and the right-hand side is quadratic in the projection operator $P$, we would like to choose $P$ in such a way that the intermediate states on the right-hand side are part of the $J^{2}=2 J(2 J+1)$ multiplet. The highest weight state in this representation is of the form

$$
|\underbrace{11 \ldots} \underbrace{2} \underbrace{2}\rangle\rangle
$$

$2 J$ times $2 J$ times

Applying the spin lowering operator $J_{-}, 2 J$ times we obtain the $J_{3}=0$ state.

This is of the form

$$
\sum_{p=0}^{2 J} N(p, 2 J-p)|\underbrace{111 \ldots}_{2 J-p} \underbrace{22 \ldots}_{p} \underbrace{i 1 \ldots}_{2 J-p} \underbrace{2 i \ldots}_{p}\rangle,
$$

where the $N$ are fixed numbers. We now choose $M_{a b}^{A} \ldots$ to be of the form (the collective index $A=p q r .$. )

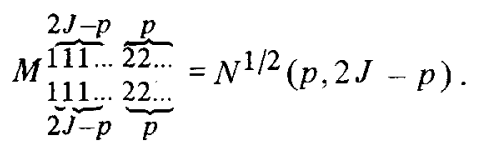

If the number of 1's and 2's do not match between upper and lower sets of indices, $M=0$. Thus

$$
\begin{aligned}
& A_{a b c \ldots, \dot{a} \dot{b} \dot{c}}^{2}(x) P_{a b c \ldots, \dot{a} \dot{b} \dot{c}} \\
& =\sum_{p} N(p, 2 J-p) A_{1}^{2} \underbrace{21 \ldots}_{2 J-p} \underbrace{22 \ldots}_{p} \underbrace{i i}_{2 J-p} \ldots
\end{aligned}
$$

This generates the $J_{3}=0$ state of the $J^{2}=J(2 J+1)$ multiplet. The left-hand side contains

$$
\begin{aligned}
& \mid \sum_{p} N(p, 2 J-p) \\
& \quad \times\left.\langle B \cdot A_{2 J-p}^{111 \ldots} \underbrace{22 \ldots}_{p}(0) B \cdot A \underbrace{1 i i \ldots}_{2 J-p} \underbrace{2 i \ldots}_{p}(x)\rangle\right|^{2} .
\end{aligned}
$$

All the intermediate states have spin $J$. We can get a state of spin $J$ from multiparticle states of lower spin as well as a single-particle state of spin $J$. In general it is not possible to separate the single-particle contribution as required for use of eq. (4). However, the inequa lity (14) is valid in general and we can consider the large $-N_{\mathrm{c}}$ limit of the correlation functions [6]. In this case the intermediate states are single glueball states and the mass appearing in eq. (4) will be the discrete point mass of the lowest spin glueball in the channel of interest. The inequality thus gives, upon using (4),

$2 m_{A B}(J) \geqslant m_{B B}(0)+m_{A A}(2 J)$.

The spins are indicated in brackets after the masses. The glueball states fall into classes. For each $J$, there is an operator of minimal dimension which can create the states. As we vary $J$ we get a "trajectory" of glueballs, If operators of dimension higher than the minimal dimension are used we get other "trajectories" Inequality (17) can be applied to the minimal case. For the minimal trajectory, we consider a mass formula of the form $a+b J^{\alpha}$ where $a$ and $b$ do not depend on the specific operators but on the trajectory as a whole, at least for large $J$ (consistent with LSZ interpretation of these operators.) Inequality (17) then gives $\alpha \leqslant 1$.

We now go back to (14). If the $P$ 's are chosen to project onto $J=0$ intermediate states on the righthand side, we get $2 m_{A B}(J) \geqslant m_{B B}(0)+m_{A A}(0)$ showing that masses in general increase with spin, i.e., we can take $\alpha \geqslant 0$ in $m=a+b J^{\alpha}$, for positive $b$.

The value of $\alpha=\frac{1}{2}$ gives a linear Regge trajectory for the glueballs. (The minimal trajectory, it seems, should be identified with the leading Regge trajectory. The formula below of variation of $m$ with the dimension of the operator supports this.) A non-relativistic rotating classical configuration has $m \sim J^{2}$ and this seems to be ruled out. In the relativistic case, one has $m \sim J$ for large $J$ and this is marginally consistent. 
We now go back to vector indices for $\operatorname{SL}(2, \mathrm{C})$ tensors. Consider

$U=A_{\mu \nu \ldots}^{a} M_{\mu \nu \ldots}^{A}, \quad V=B_{\mu \nu \ldots}^{b} M_{\mu \nu \ldots .}^{B}$.

The Cauchy-Schwarz inequality gives

$$
\begin{aligned}
& \left(A^{2} P(x) A^{2} P(0)\right)\left(B^{2} P(x) B^{2} P(0)\right) \\
& \quad \geqslant|(A B P(x) A B P(0))|^{2},
\end{aligned}
$$

where $A^{2} P=A_{\mu \nu . . .}^{a} A_{\alpha \beta \ldots}^{a} M_{\mu \nu . . .}^{A} M_{\alpha \beta \ldots}^{A}$ etc. By choosing $M$ appropriately we can project onto states of the same spin on both sides. This gives the inequality, again in the large $-N_{c}$ approximation,

$$
2 m_{A B} \geqslant m_{A A}+m_{B B} \text {. }
$$

The states connected by (20) lie on different trajectories. We can try a mass formula of the type $m=a$ $+b d^{\beta}$ where $d$ is the scaling dimension of the operator involved. Considering large $d_{B}$, with $d_{A}$ fixed, (20) gives $\beta \leqslant 1$.

Finally consider (19) where $A_{\mu \nu . .}^{a}$ is built out of a product of $F_{\mu \nu}$ 's and $B_{\mu \nu . . .}^{a}$ is the same as $A_{\mu \nu . . .}^{a}$ except for $\widetilde{F}_{\mu \nu}$ replacing one of the $F_{\mu \nu}$ 's, i.e., $B_{\mu \nu \ldots . . .}^{a}$ has opposite parity to $A_{\mu \nu \ldots . . .}^{a}$. Projecting onto the highest spin state and using large $-N_{\mathrm{c}}$ approximation (this can be done as in our discussion following (14)), we find $m_{J^{-}}$ $\geqslant m_{J^{+}}$.

In conclusiōn, we note that similar inequalities can be applied to correlations involving hybrid operators such as $F_{\mu \nu}^{a} \overline{\mathrm{q}} t^{a} \mathrm{q}$. One can relate the masses of hybrid states to pure glueball masses and multiquark states.

We acknowledge discussions with S.J. Brodsky, K. Johnson, J. Kuti, S. Meshkov, H. Neuberger, H.C. Ren and $\mathrm{C}$. Rosenzweig during the course of this investigation. This work was supported by the National Science
Foundation under grant number PHY 82-17853, supplemented by funds from the National Aeronautics and Space Administration, at the University of California, Santa Barbara.

\section{References}

[1] P.G.O Freund and Y. Nambu, Phys. Rev. Lett. 34 (1975) 1646;

H. Fritzsch and P. Minkowski, Nuovo Cimento $30 \mathrm{~A}$ (1975) 393.

[2] R.L. Jaffe and K. Johnson, Phys. Lett. B60 (1976) 201 ;

J.F. Donoghue, K. Johnson and B.A. Li, Phys. Lett. B99 (1981) 416.

[3] J.J. Coyne, P.M. Fishbane and S. Meshkov, Phys. Lett. B91 (1980) 259;

J.M. Cornwall and A. Soni, Phys. Lett. B1 20 (1983) 431.

[4] H. Gomm et al., Phys. Rev. D33 (1986) 801.

[5] M. Baker, J.S. Ball, F.Z. Chen and F. Zachariasen, Caltech preprint CALT-68-1245.

[6] G. 't Hooft, Nucl. Phys. B72 (1974) 461; E. Witten, Nucl, Phys. B160 (1979) 57.

[7] A.A. Migdal, Phys. Rep. 102 (1983) 201.

[8] D. Weingarten, Phys, Rev. Lett 51 (1983) 1830; C. Vafa and E. Witten, Nucl. Phys. B234 (1984) 173.

[9] E. Witten, Phys. Rev. Lett. 51 (1983) 2351.

[10] S. Nussinov, Phys. Rev. Lett. 51 (1983) 1081; 52 (1984) 966; E. Lieb, Phys. Rev. Lett 54 (1985) 1987.

[11] J.D. Bjorken, Proc. 1979 SLAC Summer Institute on Particle Physics;

R.L. Jaffe, J. Johnson and Z. Ryzek, MIT preprint, to be published.

[12] N.H. Christ, R. Iriedberg and T.D. Lee, Nucl. Phys. B210 [FS6] (1982) 310, 337.

[13] B.A. Berg, A. Billoire and C. Vohwinkel, Florida State University preprint FSU SCR1-86-06.

[14] S.J. Lindenbaum, Comm. Nucl. Phys. 13 (1984) 285. 